\title{
Aplicación del Análisis de Consecuencias en la Propuesta de Sistemas de Emergencia para una Planta Productora de Hidrógeno
}

\author{
Teresa Ruiz-Sánchez ${ }^{a^{*}}$, Juan Luis Francois ${ }^{\mathrm{b}}$, Pamela F. Nelson ${ }^{\mathrm{b}}$ y M. Javier Cruz-Gómez \\ ${ }^{a}$ Instituto de Energías Renovables, UNAM, Privada Xochicalco, C.P. 62580, Temixco, Morelos. \\ ${ }^{b}$ Departamento de Sistemas Energéticos, Facultad de Ingeniería, UNAM, Paseo Cuauhnáhuac No. 8532, Col. Progreso, C.P. 62550, \\ Jiutepec, Morelos, México. \\ ${ }^{c}$ Facultad de Química, UNAM, Av. Universitaria No. 3000, Col. Copilco Universidad, Del. Coyoacán, C.P. 04360, México, D.F \\ *E-mail: truizsmx@yahoo.com.mx
}

Recibido 1 de febrero de 2013, Aceptado 22 de febrero de 2013

\section{Resumen}

Actualmente, uno de los procesos que teóricamente ha demostrado ser uno de los más eficientes para la producción masiva de hidrógeno es el ciclo termoquímico Azufre-Yodo (S-I). Debido a que, este proceso de producción requiere de un gran inventario de materiales tóxicos, es necesario el diseño de sistemas de emergencia con la finalidad de proteger las instalaciones y equipos, el ambiente y la población. Unas de las consideraciones importantes para dicho diseño son los resultados obtenidos en las simulaciones de liberaciones de las sustancias involucradas en el proceso. Estas simulaciones se hicieron, previamente, con el código de computadora Phast, incluyendo tanto la fuga a través de una falla pequeña de la tubería, así como la falla catastrófica, con la finalidad de determinar la localización óptima de los equipos, así como el inventario requerido para el control de las liberaciones. Las condiciones de operación fueron tomadas de una combinación del diseño preliminar propuesto por General Atomics y las optimizadas por el Korea Advanced Institute of Science and Technology, con base en una producción de $1 \mathrm{kmol} / \mathrm{s}$ de hidrógeno. Los materiales propuestos para la neutralización de las sustancias liberadas fueron seleccionados considerando que no incrementaran el inventario de sustancias tóxicas de proceso.

Palabras clave: Ciclo S-I, sistemas de emergencia, producción de hidrógeno.

\section{Introducción}

Actualmente, el hidrógeno es ampliamente utilizado como materia prima para algunos procesos químicos tales como, producción de fertilizantes y petroquímicos, por lo cual su uso se ha incrementado. Asimismo, dada la factibilidad del empleo del hidrógeno como una alternativa energética se contempla que la demanda de éste aumente drásticamente en las próximas décadas.

Entre los métodos propuestos para la producción masiva del hidrógeno se encuentran: la electrólisis, los ciclos termoquímicos y los ciclos híbridos. De acuerdo con los trabajos reportados por Nazim Z. y Nejat [1] se muestra que, en el acoplamiento de un ciclo termoquímico con un reactor nuclear de alta temperatura proveen una ruta alternativa para la producción de hidrógeno y presentan eficiencias en el rango de 40 al $60 \%$. En las tres décadas pasadas, se han estudiado varios centenares de ciclos termoquímicos, sin embargo, sólo algunos de éstos han sido seleccionados para una evaluación técnica y económica con más detalle, entre ellos se encuentran el ciclo de azufre-yodo (S-I), el ciclo calcio-bromo (Ca-Br o UT-3), el ciclo híbrido de azufre y el ciclo híbrido de cobre-cloro $(\mathrm{Cu}-\mathrm{Cl})$.

El ciclo S-I es ampliamente investigado, por ello ya se han propuesto modificaciones al proceso propuesto por General Atomics (GA) y se han reportado eficiencias teóricas de éstas como se muestra en los trabajos de Murphy IV y O'Connell [2]. Este proceso consiste de las siguientes tres reacciones principales de acuerdo con las condiciones establecidas por GA [3]:

$$
\underset{\text { (Reacción Bunsen) }}{2 \mathrm{H}_{2} \mathrm{O}+\mathrm{SO}_{2}+\mathrm{I}_{2} \rightarrow \mathrm{H}_{2} \mathrm{SO}_{4}+2 \mathrm{HI}} \sim 120^{\circ} \mathrm{C}
$$

$$
\mathrm{H}_{2} \mathrm{SO}_{4} \rightarrow \mathrm{H}_{2} \mathrm{O}+\mathrm{SO}_{2}+1 / 2 \mathrm{O}_{2} \quad \sim 830-900{ }^{\circ} \mathrm{C}
$$

(Descomposición ácido sulfúrico, $\mathrm{SA}$ )

$$
\begin{gathered}
2 \mathrm{HI} \rightarrow \mathrm{H}_{2}+\mathrm{I}_{2} \sim 300-450{ }^{\circ} \mathrm{C} \\
\text { (Descomposición ácido yodhírico, HIx's) }
\end{gathered}
$$

Como puede observarse en las ecuaciones de arriba, la mayoría de las sustancias involucradas en el ciclo S-I son consideradas como peligrosas. Actualmente, las regulaciones y normativas de la industria química no establecen como requisito un análisis de consecuencias ni el diseño de sistemas para el control liberaciones. Sin embargo, se considera que dado el efecto que un evento de fuga 
de los materiales incluidos en este proceso tendrían un gran impacto en el medio ambiente y en la población cercana es necesario contar con sistemas que ayuden a disminuir estas consecuencias adversas aun cuando la probabilidad de que ocurra una fuga sea pequeña.

Para determinar las dimensiones de las nubes con concentraciones tóxicas debido a una liberación de materiales peligros (compuestos sulfurados y de yodo) se realizaron simulaciones con el código de computadora Phast 6.1 [4]. Los resultados obtenidos para determinar las zonas de afectación de las liberaciones fueron ya reportados en trabajos previos [5]. En particular, en este trabajo se presentan las propuestas para los sistemas de emergencias necesarios para el control de liberación de compuestos sulfurados (ácido sulfúrico, di y trióxido de azufre), ácido yodhídrico y yodo.

\section{Condiciones de proceso base y simulaciones}

Las nuevas tecnologías para la producción de hidrógeno requieren una evaluación de aspectos de seguridad basada en las aproximaciones disponibles.

Phast es un programa de cómputo que combina diferentes modelos para poder realizar un análisis de la secuencia del accidente desde la liberación del material hasta la explosión/combustión o su dispersión. El modelado de las consecuencias en el programa Phast involucra el progreso de un potencial incidente, desde la liberación inicial (modelo de descarga) hasta su completa dispersión, incluyendo la condensación de la nube y la evaporación del charco, así como la liberación de energía por fuego $o$ explosión (materiales inflamables).

Una característica de Phast es que no requiere demasiados datos de entrada ni un excesivo tiempo de cálculo, lo cual lo hace compatible con los requerimientos para los estudios de riesgo y de seguridad, por ello éste es ampliamente utilizado en el análisis de riesgos de instalaciones industriales.

Los datos de entrada para las simulaciones deben considerar como base las condiciones de proceso (presión, temperatura, flujos), las condiciones atmosféricas representativas de la zona donde la planta química será instalada, y los diámetros propuestos.

\subsection{Condiciones de Proceso del Ciclo Termoquímico S-I}

El ciclo termoquímico S-I fue desarrollado por
General Atomics Corporation en la década de 1980's. Sin embargo, se requiere de algunos desarrollos antes de que este ciclo pueda ser utilizado para la producción masiva del hidrógeno. Así las simulaciones de este estudio fueron realizadas con base en la reacción Bunsen (ver la ecuación 1) y de datos experimentales publicados. Lee B.J. et al. Optimizaron las condiciones de proceso para obtener una solución de ácido yodhídrico (HIx's) por encima de la mezcla azeotrópica $[6,7]$. Los flujos fueron normalizados para una producción de $1 \mathrm{kmol} / \mathrm{s}$ de hidrógeno, así mismo, las relaciones estequiométricas $\mathrm{y}$ parámetros de operación usados en este trabajo son mostrados en la Figura 1, donde $\mathrm{x}=4, \mathrm{~m}=5 \mathrm{y} \mathbf{n}=$ 6.

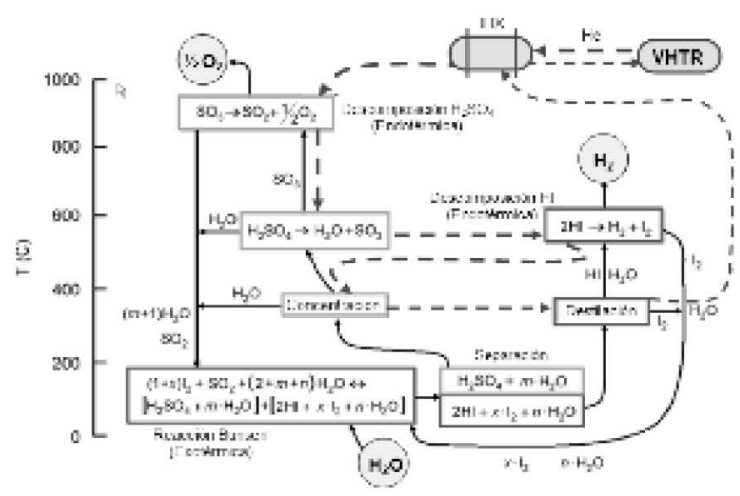

Figura 1. Esquema general de las principales reacciones de proceso del ciclo S-I. Adaptado [6]

\subsection{Información Complementaria para la Simulación}

Las condiciones atmosféricas consideradas en este estudio fueron las representativas del Golfo de México: temperatura promedio: $26{ }^{\circ} \mathrm{C}$ y humedad relativa: $79 \%$. Tres clases de estabilidad atmosférica fueron evaluadas: la clase A (muy inestable), la clase D (neutral) y la clase F (muy estable). Estas clases fueron modeladas con diferentes velocidades de viento conformando los siguientes pares climáticos: $1.5 \mathrm{~m} / \mathrm{s}-\mathrm{F}, 2 \mathrm{~m} / \mathrm{s}-\mathrm{D}, 5$ $\mathrm{m} / \mathrm{s}-\mathrm{D}$ y $1.5 \mathrm{~m} / \mathrm{s}-\mathrm{A}$.

Las simulaciones fueron hechas tomando en cuenta un diámetro de la fuga de $2.54 \mathrm{~cm}$ para los materiales tóxicos (fuga más probable por falla de empaques, uniones, etc.) y de $101.6 \mathrm{~cm}$ para las liberaciones de hidrógeno (falla catastrófica de la tubería de mayor diámetro). El inventario del material a la descarga estuvo basado en el flujo de material que puede liberarse en 10 minutos. 


\section{Diseño de sistemas de emergencia.}

Para el diseño de los sistemas de emergencia deben considerarse tanto los resultados de las simulaciones de las liberaciones accidentales de los materiales, como las propiedades de éstos. Así, en las siguientes subsecciones de este documento se definen especificaciones para la propuesta de estos sistemas.

\subsection{Aplicación de los Resultados de las Simulaciones}

La relevancia de los resultados de las simulaciones con Phast para el diseño de los sistemas de emergencia, es básicamente la localización de los sensores y la distribución de los equipos. Así con los resultados de las simulaciones podemos determinar el número óptimo de sensores y su ubicación considerando las variaciones en la velocidad del viento $y$ otras condiciones atmosféricas. Por otro lado, las dimensiones de las nubes que se forman a partir de la liberación accidental de las sustancias tóxicas son necesarias para definir la capacidad y distribución del equipo incluido en los sistemas de emergencia. Así, una buena localización del equipo podría evitar que éste se dañe debido a la energía generada por la expansión de los gases en las liberaciones de los materiales.

La distancia alcanzada por las nubes cuando el modelo de expansión es predominante (la dirección del viento no influye en el modelo de dispersión) puede ser utilizada para determinar la localización de los sensores de gas (especialmente en tuberías de gran longitud), para mejorar la distribución de la planta (layout) y del equipo de sistemas de emergencias. Estas distancias, alcanzadas en 10s o menos, deben ser comparadas en los casos de falla probable y falla catastrófica para definir el área incluyente, tal como se muestra en la Figura 2 en donde podemos observar la vista superior de una fuga de dióxido de azufre. Los resultados de las simulaciones para una fuga probable ya fueron reportados en un trabajo previo [5]. Así, considerando la geometría de la falla probable en la Figura 2, por lo menos un sensor de dióxido de azufre deberá instalarse a cada $8 \mathrm{~m}$ de longitud de tubería de la sección de descomposición de ácido sulfúrico a una distancia perpendicular de $70 \mathrm{~m}$. Sin embargo, el inventario de materiales neutralizantes deberá considerarse para una falla catastrófica.

Debido a que actualmente no existen sensores comerciales para todas las sustancias tóxicas de proceso, se propone lo siguiente:

- La distribución de planta ha sido definida con base en las reacciones en las diferentes secciones de proceso, es decir, las diferencias en los compuestos de materiales de proceso debe ser considerada. Esto dado que los compuestos sulfurados reaccionan violentamente con el agua y los compuestos de yodo no reaccionan con ésta, o su grado de reacción es menor. Dada esta característica se propone utilizar el agua como medio para precipitar el yodo, la distribución de la planta debe tomarse como base en la separación de las reacciones de proceso y no sólo la eficiencia energética.

- La sección de descomposición de ácido sulfúrico debe ser monitoreada con sensores comerciales de dióxido de azufre, tales como los distribuidos por Dräger Safety Inc.

- La sección de descomposición de ácido yodhídrico debe ser monitoreada con sensores de yodo; sin embargo, actualmente no existen sensores comerciales para éste. No obstante, algunas investigaciones han demostrado la viabilidad para desarrollarlos [8].

Otra de las aplicaciones de las simulaciones con Phast es utilizar la reducción en la dispersión de la nube cuando se modifica la clase de estabilidad atmosférica considerando como una aproximación en la reducción del material liberado debido a la actuación de los sistemas de emergencia.

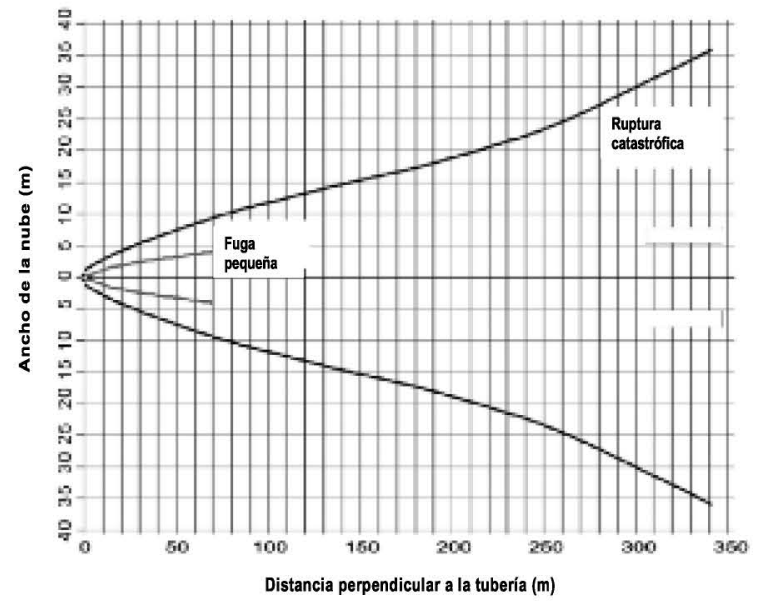

Figura 2. Vista superior de la nube de dióxido de azufre a los 10 segundos para una falla catastrófica y una fuga pequeña

\subsection{Diseño del sistema de control de liberación de compuestos de yodo}

Para el control de la liberación de la solución de ácido yodhídrico y yodo se propuso un sistema de aspersión de agua. Lo anterior, dado que el agua presenta la característica de diluir el ácido y no ser 
miscible con el yodo. Adicionalmente, la aspersión de agua produce un movimiento vertical, lo cual reduce la estabilidad en esta dirección.

Las principales consideraciones que se hicieron para tomarse como base de diseño fueron las siguientes:

- Se requieren 4 moles de agua para disolver una mol de ácido yodhídrico.

- Se incluyó el calor de disolución.

- Disipación de calor de la mezcla considerando la temperatura final más alta para la dispersión de una nube en la sección HIx.

- Temperatura ambiental de $25^{\circ} \mathrm{C}$.

- Flujo másico mayor de yodo en cualquier sección de proceso: $2.86 \mathrm{kmol} / \mathrm{s}$ de $\mathrm{HI}, 1.1 \mathrm{kmol} / \mathrm{s}$ de $\mathrm{I}_{2}$ y $1.53 \mathrm{kmol} / \mathrm{s} \mathrm{de} \mathrm{H}_{2} \mathrm{O}$.

El flujo mínimo de agua se determinó con base en el flujo requerido para la disipación de calor generado por la mezcla, cuya temperatura final de la nube es de $203{ }^{\circ} \mathrm{C}$, así como el calor generado por la disolución del HI. Para ello, se obtuvieron las capacidades caloríficas y el calor de disolución mediante el cálculo de propiedades de lo materiales que tiene el programa Phast, usando como base los coeficientes definidos en la referencia [9]. Los datos obtenidos fueron verificados por las ecuaciones propuestas por Kobe [10].

El calor de disipación se calculó con la integral de la capacidad calorífica en el rango de temperaturas establecido $\left(\mathrm{T} 2=25{ }^{\circ} \mathrm{C}, \mathrm{T} 1=203\right.$ $\left.{ }^{\circ} \mathrm{C}\right)$, de acuerdo con la función:

$$
Y=A+B\left[\frac{C / T}{\sinh (C / T)}\right]^{2}+D\left[\frac{E / T}{\cosh (E / T)}\right]^{2}
$$

Donde Y representa la capacidad calorífica. Los valores de las propiedades de los materiales, el flujo de calor generado por la disolución y disipación son mostrados en la Tabla 1.

Así para la disipación de este calor se requiere de $3.6 \mathrm{~kg} / \mathrm{s}$ de agua $(198.84 \mathrm{~mol} / \mathrm{s})$.

Tabla 1. Propiedades de los compuestos de yodo y calor generado

\begin{tabular}{ccc}
\hline & & $\begin{array}{c}\text { Calor } \\
\text { generado }\end{array}$ \\
\hline $\begin{array}{c}\text { Calor disolución HI } \\
\text { Capacidad Calorífica } \\
\mathrm{HI}\end{array}$ & $-83.33 \mathrm{~kJ} / \mathrm{mol} \mathrm{HI}$ & $-238324 \mathrm{~kJ} / \mathrm{s}$ \\
$\begin{array}{c}\text { Capacidad Calorífica } \\
\mathrm{I}_{2}\end{array}$ & $0.02930 \mathrm{~kJ} / \mathrm{mol}^{\circ} \mathrm{C}$ & $-14832.2 \mathrm{~kJ} / \mathrm{s}$ \\
& $0.03713 \mathrm{~kJ} / \mathrm{mol}^{\circ} \mathrm{C}$ & $-7229.21 \mathrm{~kJ} / \mathrm{s}$ \\
\hline & Total & $-260385 \mathrm{~kJ} / \mathrm{s}$ \\
\hline
\end{tabular}

Por otro lado, para la disolución de $\mathrm{HI}$ se requiere un flujo de $205.92 \mathrm{~kg} / \mathrm{s}$ de agua $(11440$ $\mathrm{mol} / \mathrm{s}$ ). Adicionalmente, si consideramos que una mol de agua se adhiere a una mol de yodo se requiere de un flujo de $19.8 \mathrm{~kg} / \mathrm{s}$ de agua $(1100$ $\mathrm{mol} / \mathrm{s})$. Así, los requerimientos de flujo de agua total del sistema son de $229.3 \mathrm{l} / \mathrm{s}$. Si se toma en cuenta que este sistema debe funcionar el tiempo máximo de la fuga (determinado en las simulaciones como de 10 minutos), entonces el tanque de almacenamiento de agua para este sistema deberá contener como mínimo una capacidad de almacenamiento de $137.6 \mathrm{~m}^{3}$.

El sistema propuesto está comprendido básicamente por el tanque de almacenamiento de agua, una bomba con su redundancia que será arrancada con la señal combinada de presencia de yodo (monitoreada por el sensor, concentración de $6 \mathrm{ppm})$ con baja presión en el equipo/sección de tubería más cercano al orificio de fuga. Las tuberías del sistema de aspersión serán seccionadas por tramo de tubería de la longitud determinada por el ancho de la nube de acuerdo a las simulaciones con Phast. Adicionalmente, cada equipo tendrá una sección en el sistema de aspersión. La ubicación del sensor de yodo que detecta la presencia de éste enviará también una señal de apertura a la válvula solenoide correspondiente a la sección de tubería de aspersión, para que se dirija sólo a esta área el flujo de agua. El esquema simplificado de este sistema se muestra en la Figura 3.

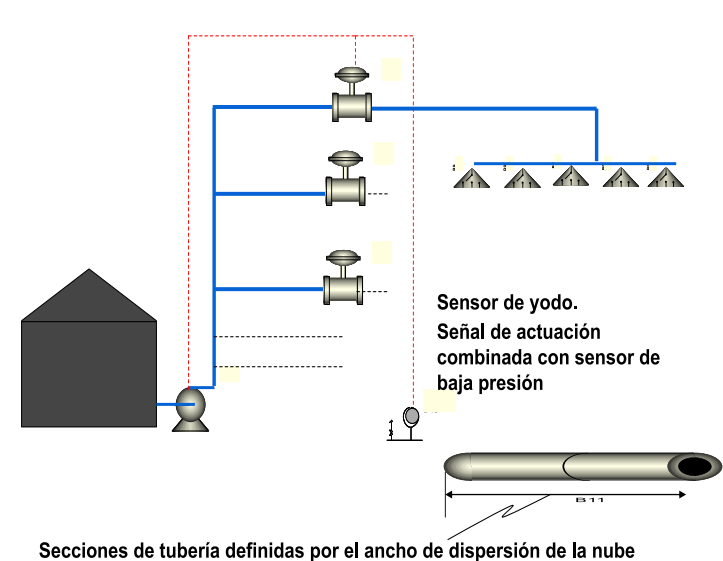

Figura 3. Esquema simplificado del sistema de control de liberación de la mezcla $\mathrm{HI}-\mathrm{I}_{2}-\mathrm{H}_{2} \mathrm{O}$

\subsection{Diseño del sistema de control de liberación de compuestos sulfurados}

Para el control de la liberación de compuestos sulfurados se propone un sistema de aspersión combinado de bicarbonato de sodio y agua tibia (40 
$\left.{ }^{\circ} \mathrm{C}\right)$. Lo anterior, dado que el ácido sulfúrico presenta una reacción altamente exotérmica al diluirse en agua, por lo cual se propone que con la reacción de bicarbonato $\mathrm{y}$ agua se forme el hidróxido de sodio antes de que el agua tenga contacto con el ácido. El empleo del bicarbonato de sodio se consideró como una buena alternativa ya que éste presenta las características de ser barato y no tóxico.

Las principales consideraciones que se hicieron para tomarse como base de diseño fueron las siguientes:

- Eficiencia de reacción de neutralización con bicarbonato del $80 \%$.

- Se incluyó el calor de disolución.

- Disipación de calor de la mezcla considerando la temperatura final más alta para la dispersión de una nube en la sección ácido sulfúrico.

- Temperatura ambiental de $25^{\circ} \mathrm{C}$.

- Flujo másico mayor de ácido sulfúrico en

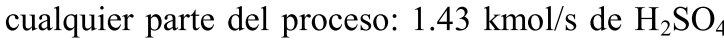
y $1.53 \mathrm{kmol} / \mathrm{s} \mathrm{de} \mathrm{H}_{2} \mathrm{O}$.

Los requerimientos de $\mathrm{NaHCO}_{3}$ se determinaron con la eficiencia de la reacción ya definida, lo cual de como resultado una cantidad de $150.1 \mathrm{~kg} / \mathrm{s}$ de bicarbonato $(1.79 \mathrm{kmol} / \mathrm{s})$.

El flujo mínimo de agua se determinó con base en el flujo requerido para la disipación de calor generado por el enfriamiento de la mezcla (considerando el ácido sulfúrico como componente puro), cuya temperatura final de la nube es de 335 ${ }^{\circ} \mathrm{C}$, así como el calor generado por la disolución de dicho ácido con un exceso de agua. Para ello, se obtuvieron la capacidad calorífica y el calor de disolución mediante el cálculo de propiedades de los materiales que tiene el programa Phast, usando como base los coeficientes definidos en la referencia [9]. Cabe mencionar que se consideró la mezcla que contiene ácido sulfúrico y no la que contiene óxidos de azufre, dado que éstos poseen una capacidad calorífica menor a la del ácido.

El calor de disipación se calculó con la integral de la capacidad calorífica en el rango de temperaturas establecido $\left(\mathrm{T} 2=25^{\circ} \mathrm{C}, \mathrm{T} 1=335\right.$ ${ }^{\circ} \mathrm{C}$ ), de acuerdo con la función establecida en la ecuación (4).

Los valores de las propiedades del ácido sulfúrico, el flujo de calor generado por la disolución y disipación son mostrados en la Tabla 2.

Así para la disipación de este calor se requiere de $370.3 \mathrm{~kg} / \mathrm{s}$ de agua $(20.5 \mathrm{kmol} / \mathrm{s})$.

Por otro lado, para la disolución con $\mathrm{NaHCO}_{3}$ considerando una solubilidad del $10 \%$ se requiere de un flujo de $290 \mathrm{~kg} / \mathrm{s}$ de agua $(16.11 \mathrm{kmol} / \mathrm{s})$. Sin embargo, la reacción de disolución de bicarbonato es endotérmica lo cual ayudaría a reducir el calor
Tabla 2. Propiedades del ácido sulfúrico y calor generado

\begin{tabular}{ccc}
\hline & & $\begin{array}{c}\text { Calor } \\
\text { generado }\end{array}$ \\
\hline Calor disolución & $-96.19 \mathrm{~kJ} / \mathrm{mol} \mathrm{H}_{2} \mathrm{SO}_{4}$ & $-137551.7 \mathrm{~kJ} / \mathrm{s}$ \\
$\begin{array}{c}\text { Capacidad } \\
\text { Calorífica }\end{array}$ & $\begin{array}{c}18742.70768 \mathrm{~kJ} / \mathrm{mol} \\
\mathrm{H}_{2} \mathrm{SO}_{4}\end{array}$ & $-26802072 \mathrm{~kJ} / \mathrm{s}$ \\
\hline
\end{tabular}

Total $-26939624 \mathrm{~kJ} / \mathrm{s}$

de disolución del ácido. Por esto se considera que dado que el exceso de agua para disipar el calor es menor que para realizar la disolución entonces se tiene que los requerimientos de flujo de agua total del sistema es de $370.3 \mathrm{l} / \mathrm{s}$ de agua. Si se considera que este sistema debe funcionar el tiempo máximo de la fuga (determinado en las simulaciones como de 10 minutos), entonces el tanque de almacenamiento de agua para este sistema deberá contener como mínimo una capacidad de almacenamiento de $222.2 \mathrm{~m}^{3}$.

\section{Conclusiones}

Los resultados de las simulaciones con Phast pueden ser utilizados, en el diseño de sistemas de emergencia, para la determinación de la localización óptima de los sensores de gas, la distribución general del equipo en la planta y las estrategias que incluyen la zona de exclusión (como se emplea en la regulación nuclear). Adicionalmente, al variar las clases de estabilidad atmosférica por condiciones de menor estabilidad se puede obtener una buena aproximación en la reducción de la distancia alcanzada por las nubes tóxicas debido a la actuación de los sistemas de emergencia.

Los sistemas de emergencia propuestos no incrementan el inventario de sustancias peligrosas incluidas en el proceso debido a que se utiliza agua y bicarbonato de sodio. El inventario de estas nuevas sustancias considera el flujo másico liberado durante diez minutos en una falla catastrófica (la menos probable), por lo cual cualquier liberación menor de las sustancias tóxicas estará completamente controlada mediante la actuación de los sistemas de emergencia.

Los resultados de la dispersión de la nube de yodo pueden ser considerados como una alternativa para determinar la zona de exclusión de la planta química de manera similar como lo establecido en las regulaciones para las instalaciones nucleares [11]. Lo anterior dado que las simulaciones de los materiales tóxicos fueron realizadas considerando un diámetro de fuga de $2.54 \mathrm{~cm}$, es decir, para el 
evento de fuga más probable. Asimismo, se tendrían que considerar los tiempos en los cuales se alcancen las diferentes distancias de interés tales como población cercana y edificios de personal para el diseño de los tiempos de respuesta en los planes de emergencia.

En el caso de utilizarse como fuente de calor para las reacciones de descomposición de los ácidos, un reactor nuclear de alta temperatura, el personal de cada instalación deberá contar con el equipo de protección y medidas de seguridad necesarias para el manejo tanto de sustancias tóxicas como de alguna liberación radiactiva.

\section{Agradecimientos}

Se hace un agradecimiento especial al Consejo Nacional de Ciencia y Tecnología (CONACYT) por la beca de doctorado otorgada a T. RuizSánchez, con la cual se hizo posible este trabajo.

\section{Referencias}

1. Muradov, N. Z.; Veziroğlu; T. N. Int. J. Hydrogen Energy, 2008, 33, 6804-6839.

2. Murphy, IV; J., E.; O'Connell, J. P. Int. J. Hydrogen Energy, 2012, 37, 4002- 4011.

3. Brown, L. C.; Funk, J. F.; Pickard, P.; Marshall, A.; Showalter, S. K. High efficiency generation of hydrogen fuels using nuclear power - Final Techical Report, General Atomics Report GA-A24285, Rev. 01, December 2003.

4. Phast 6.1 Professional, Process Hazard Analysis Software Tool, Norway: DNV, 1999.

5. Ruiz-Sánchez T.; Nelson, P. F. ; Francois, J. L., CruzGómez, M. J. Int. J. Hydrogen Energy, 2012, 37, 6965 6975.

6. Lee, B.J.; NO, H. Ch.; Yoon, H. J.; Jin, H. G.; Kim, Y. S.; Lee, J. I. Int. J. Hydrogen Energy, 2009, 34, 2133-2143.

7. Lee, B.J.;, NO, H. Ch.; Yoon, H. J.; Kim, Y. S.; Kim, E. S. Int. J. Hydrogen Energy, 2008, 33, 2200-2210.

8. Dulsev F. Sensor \& Act. B: Chemicals, 2008, 129, 171-175.

9. Daubert, T. E.; Danner, R. Physical and Thermodynamic Properties of Pure Chemicals. Data Compilation, DIPPR (Design Institute for Physical Property Data), Estados Unidos; 1999.

10. Kobe, K. A. Petroleum Refiner, 1949-1954

11. United States Nuclear Regulatory Commission (USNRC), 10 CFR Part. 100.11, Determination of exclusion area, low population zone, and population center distance, http://www.nrc.gov/reading-rm/doc-

collections/cfr/part100/part100-0011.html (accesado el 20 de octubre de 2011). 